\title{
Vortical structures in a shallow wake of the flow around a confined cylinder at $R e=3750$
}

\author{
Egor Palkin ${ }^{1,2}$, and Rustam Mullyadzhanov ${ }^{1,2}$ \\ ${ }^{1}$ Institute of Thermophysics SB RAS, 630090, Novosibirsk, Russia \\ ${ }^{2}$ Novosibirsk State University, 630090, Novosibirsk, Russia
}

\begin{abstract}
We study the flow over a cylinder placed between two parallel rigid walls using Large-eddy simulations. The Reynolds number based on the inflow velocity and diameter of the cylinder is 3750 corresponding to the subcritical regime with laminar separation. The comparison of the timeaveraged flow patterns shows very good agreement with experiments. The unsteadiness of the recirculating bubble features the low-frequency modulation observed for the first time.
\end{abstract}

\section{Introduction}

Flows over obstacles in a duct are common in many engineering applications such as cooling systems, bridge piers, heat exchangers, building sections, junctions in wing-body and turbine blade-rotor systems, among others. In such configurations a horseshoe vortex system appears prior to a bluff body increasing the local shear stress and heat transfer. Figure 1 demonstrates the changes introduced by the flow confinement comparing the flow over an infinite cylinder (left) and with side walls (right) at close Reynolds numbers. Aside from the horseshoe vortex, the wake becomes dominated by streaky structures. In the present work we focus our attention on the basic time-averaged characteristics of the flow and unsteady behaviour of the recirculating bubble.
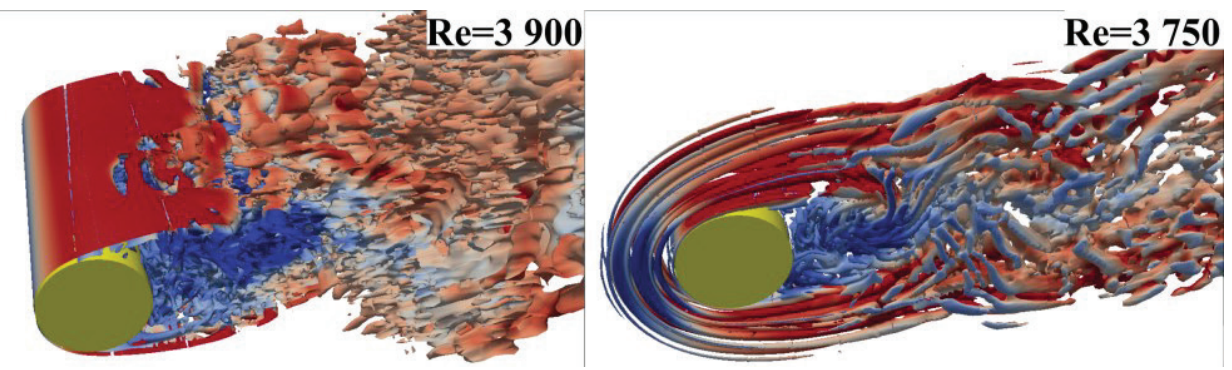

Fig. 1. The isosurface of the $Q$-criterion identifying vortical structures colored with axial velocity. Left: unconfined cylinder (infinite along the spanwise direction) from our previous results [1] at $R e=$ 3900; right: quasi-2D present configuration at $R e=3750$ with $H / D=0.4$, where $H$ is the distance between parallel walls and $D$ is the diameter of the cylinder. 


\section{Computational details}

The Large-eddy simulation (LES) was carried out with the unstructured finite-volume computational code T-FlowS. The filtered Navier-Stokes and continuity equations for incompressible fluid are closed by the dynamic Smagorinsky subgrid-scale model. The diffusion and convection terms in the momentum equations are discretized by the secondorder central-difference scheme, whereas the time-marching is performed using a fullyimplicit three-level time scheme. The velocity and pressure are coupled with the SIMPLE algorithm.

The computational domain is shown in Fig. 2. We study the flow over a circular cylinder fixed between a pair of side walls at the Reynolds number of $R e=3750$ based on the bulk inflow velocity $U_{b}$ and cylinder diameter $D$. The domain represents a rectangle of the size $x \times y \times z=29 D \times 20 D \times H$ leading to $8.7 \mathrm{mln}$ cells grid, where $H=0.4 D$ is the distance between parallel walls corresponding to $z=0$ and $z=0.4 D$ planes. The inflow is provided by a steady parabolic laminar profile, while no-slip conditions are set at all walls. The total computational time was around $t U_{\infty} / D=10^{3}$ with a timestep $\Delta t U_{\infty} / D=0.0025$. The statistics was gathered for 500 non-dimensional time units.

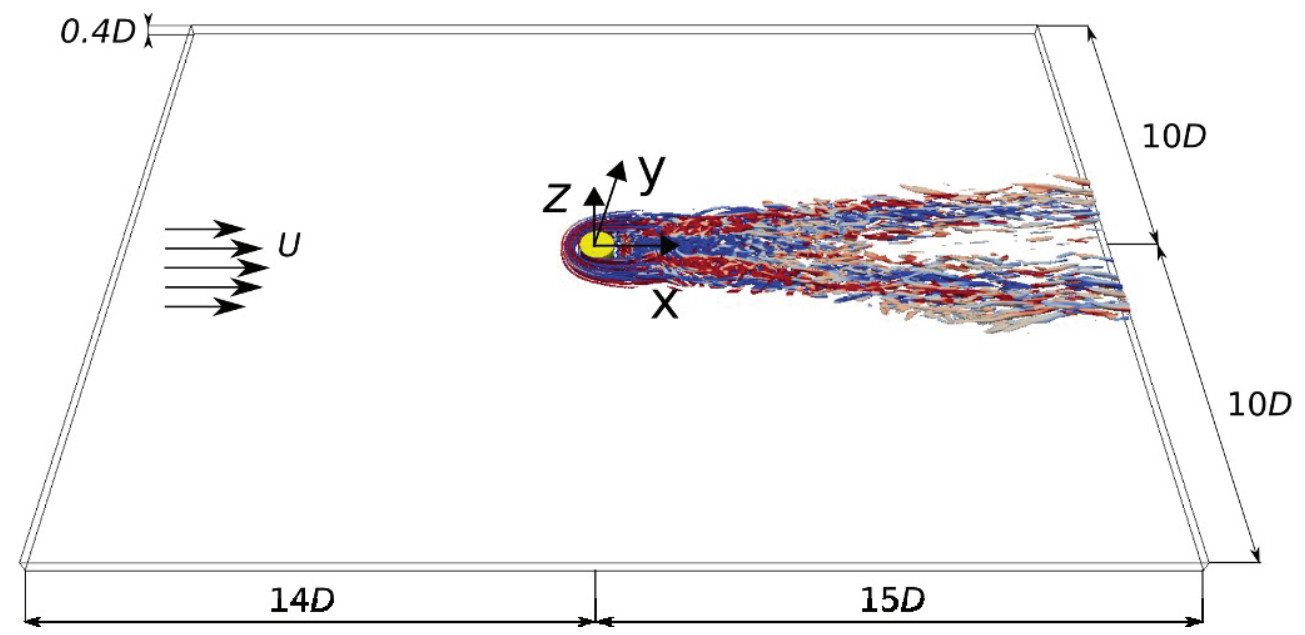

Fig. 2. Computational domain. Surface on the left corresponds to the inflow boundary condition, the outflow is on the right. All other surfaces are rigid walls, yellow surface denotes the cylinder.

\section{Results}

At this low Reynolds numbers the laminar inflow turbulizes only in the wake with a slow transition (growing of disturbances) in the shear layer. The typical vortical structure in the near wake represents a meandering elongated vortex, Fig. 1. Further downstream one can still recognize elongated coherent structures. The presence of walls strongly affects dynamics of the flow resulting in the change of time-averaged characteristics. Figure 3 (left) shows the comparison of the time-averaged axial velocity and streamlines for the confined (present) case in the mid-plane $(z=0.5 H=0.2 D)$ and without walls from previous simulations [1]. While the pattern is the same, the recirculating bubble shrinks and becomes narrower in the presence of walls. Turbulent kinetic energy is shown in Fig. 3 very close to the wall denoting the footprint of a slightly fluctuating horseshoe vortex appearing in front 
of the cylinder [2]. The streamlines near the wall differ significantly from those at the midplane pointing at strong three-dimensionality of the flow.



Fig. 3. Left: Time-averaged axial velocity for the present confined case of the flow over a cylinder at $z=0.5 H=0.2 D$ (top) and the one without walls from previous simulations [1] (bottom). Right: turbulent kinetic energy and streamlines near the wall.

Figure 4 (top) presents the streamlines from particle image velocimetry (PIV) measurements at $R e=4000$ in $x-z$ plane at $y=0$ before and after the cylinder [2] showing time-averaged vortical structure of the flow. In the bottom of Fig. 4 we compare it with LES results, namely the time-averaged vertical velocity fluctuations field and streamlines indicating very good comparison with experiments. The position of main vortices is well reproduced.

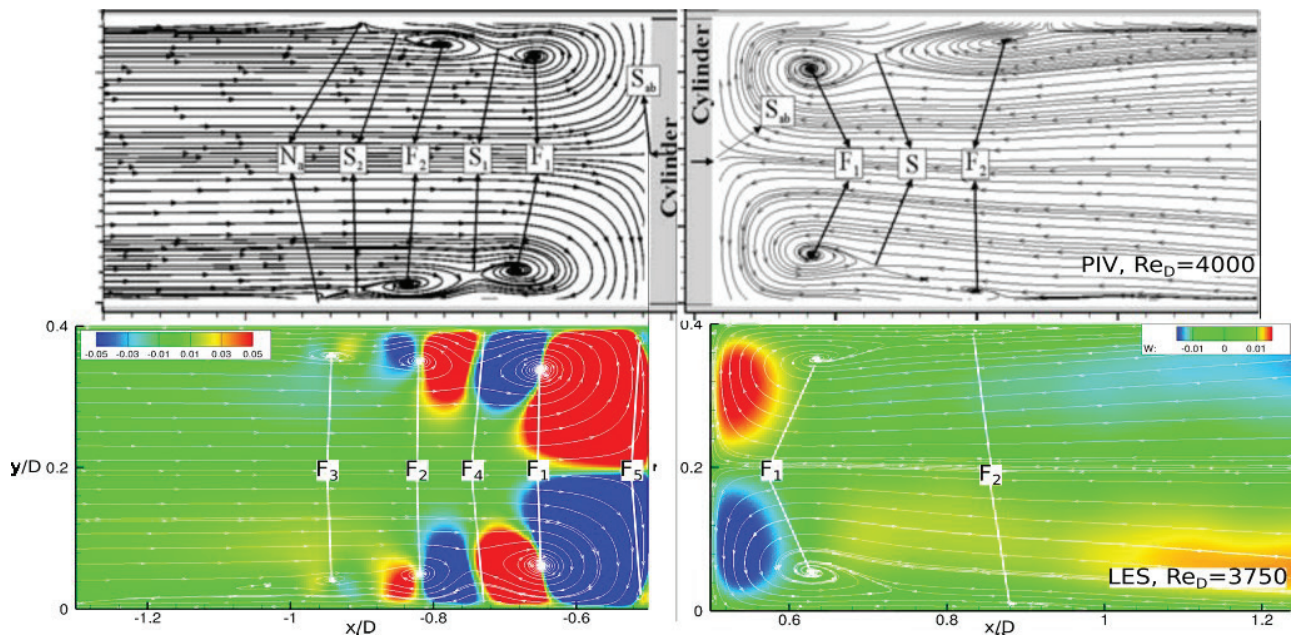

Fig. 4. Top: time-averaged streamlines in $x-z$ plane and $y=0$ from PIV measurements [2]. Bottom: vertical velocity field and corresponding streamlines from LES.

Figure 5 shows the comparison of the instantaneous velocity signal in the shear layer at $z=0.5 \mathrm{H}$. The filtered signal reveals the existence of low-frequency oscillations of the recirculation zone. The non-dimensional period is around $28.6 D / U_{b}$ corresponding to the frequency $f=0.035 U_{b} / D$. These results are in agreement with numerical simulations $[1,3]$ of unconfined cylinder where such modulations have also been detected. 

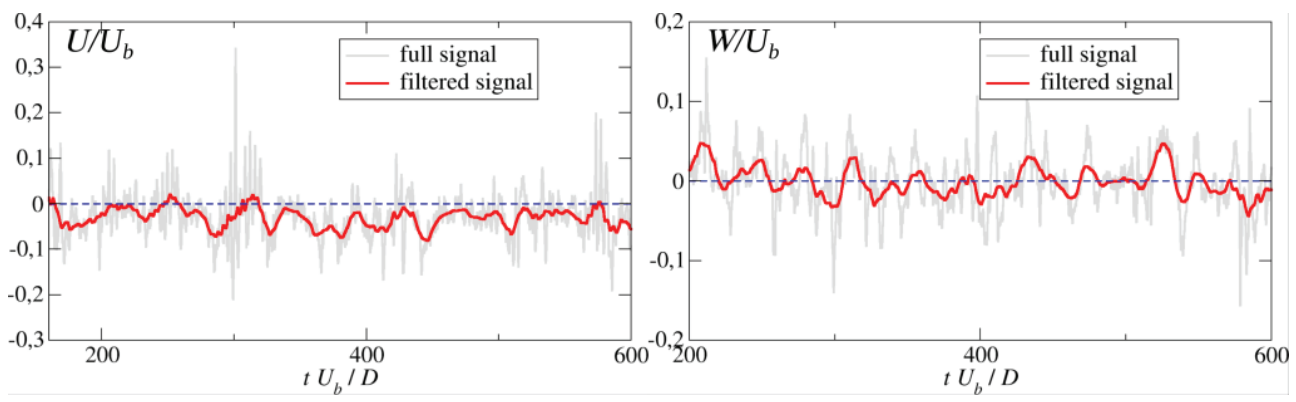

Fig. 5. Left: Instantaneous signal of axial velocity in the point $(x / D, y / D, z / D)=(0.5,0.5,0.2)$. Right: The same for the vertical velocity component.

We have presented the results of Large-eddy simulations of the confined flow over a cylinder at $R e=3750$. The direct comparison with PIV experiments showed very good agreement of the position of main time-averaged vortices. We find that the recirculating bubble manifests low-frequency oscillations similar to the unconfined case $[1,3]$. Further work will be devoted to studying the elongated vortical structures found also in confined turbulent jets [4-7] and their role in heat transfer across the channel.

This work is funded by the Russian Science Foundation grant No. 14-19-01685. The computational resources are provided by Siberian Supercomputer Center SB RAS and Supercomputing Center of the Novosibirsk State University.

\section{References}

1. E. Palkin, R. Mullyadzhanov, M. Hadziabdic, K. Hanjalic, Flow Turbul. Combust., appeared online (2016), doi:10.1007/s10494-016-9772-z

2. N.A. Ozturk, A. Akkoca, B. Sahin, Exp. Fluids 44 (6), 1001-1014 (2008)

3. O. Lehmkuhl, I. Rodriguez, R. Borrell, A. Oliva, Phys. Fluids, 25 (8), 085109 (2013)

4. R. Mullyadzhanov, B. Ilyushin, K. Hanjalic, Int. J. Heat Fluid Flow, 56, 284-289 (2015)

5. K. Hanjalic, R. Mullyadzhanov, Phys. Fluids, 27 (3), 031703 (2015)

6. R. Mullyadzhanov, B. Ilyushin, M. Hadziabdic, K. Hanjalic, In: Direct and Large-Eddy Simulation IX, Springer, Netherlands, (2015).

7. M.V. Shestakov, R.I. Mullyadzhanov, M.P. Tokarev, D.M. Markovich, J. Eng. Thermophys. 25, 159 (2016)

8. SThermophys., 25 (2), 159-165 (2016) 